\title{
Numerical Analysis of Chloride Diffusion in Concrete with Time Varying Coefficient Based on the ADI Method
}

\author{
Yu Bai ${ }^{1,2, ~ *, ~ M e n g j i e ~ T a n g ~}{ }^{1}$, Fei Hou ${ }^{1}$, Jun Dong ${ }^{2,3}$ \\ ${ }^{1}$ Department of Mathematics, Beijing University of Civil Engineering and Architecture, Beijing, China \\ ${ }^{2}$ Beijing Key Laboratory of Functional Materials for Building Structure and Environment Remediation, Beijing University of Civil \\ Engineering and Architecture, Beijing, China \\ ${ }^{3}$ School of Civil and Transportation Engineering, Beijing University of Civil Engineering and Architecture, Beijing, China
}

\section{Email address:}

baiyu@bucea.edu.cn (Yu Bai)

${ }^{*}$ Corresponding author

\section{To cite this article:}

Yu Bai, Mengjie Tang, Fei Hou, Jun Dong. Numerical Analysis of Chloride Diffusion in Concrete with Time Varying Coefficient Based on the ADI Method. Mathematical Modelling and Applications. Vol. 2, No. 1, 2017, pp. 1-7. doi: 10.11648/j.mma.20170201.11

Received: February 15, 2017; Accepted: March 3, 2017; Published: March 21, 2017

Abstract: In this paper, a two-dimensional finite difference numerical model with time varying coefficient using Alternating Direction Implicit Method (ADI) is developed to predict Chloride diffusion in concrete. This model is proved to be unconditionally stable and has higher accuracy. And a numerical example is given to show the effectiveness of this model.

Keywords: Chloride Ion, Time Varying Coefficient, ADI Method, Stable, Convergent

\section{Introduction}

The diffusing mechanism of Chloride ion in reinforced concrete structures is very complex. Generally, it includes diffusion effect, capillary effect, permeation effect, chemical migration effect and their combinations, and the diffusion effect plays a leading role. Model of Chloride ion diffusion equation is established mainly based on the Fick's second law [1] which can combine Chlorine ion concentration with diffusion coefficient and diffusion time. Chloride ion diffusion coefficient is often considered to be a constant when we calculate the numerical model of the Chloride ion diffusion. Considering the two-dimensional diffusion of Chloride ion under actual conditions, the equation can be rewritten as [2]

$$
\frac{\partial C}{\partial t}=D\left(\frac{\partial^{2} C}{\partial x^{2}}+\frac{\partial^{2} C}{\partial y^{2}}\right)
$$

where $C$ is the Chloride ion concentration, $D$ is the constant diffusion coefficient. With the initial conditions

$$
C(x, y, 0)=C_{0},(0<x, y<\infty)
$$

and the boundary conditions

$$
\begin{aligned}
& C(0, y, t)=C(x, 0, t)=C_{s} \\
& C(\infty, y, t)=C(x, \infty, t)=C_{0}
\end{aligned}
$$

the analytical solution [3] of Eq.(1)-Eq.(3) is

$$
\begin{aligned}
& C(x, y, t) \\
& =C_{0}+\left(C_{s}-C_{0}\right)\left[1-\operatorname{erf}\left(\frac{x}{2 \sqrt{D t}}\right) \operatorname{erf}\left(\frac{y}{2 \sqrt{D t}}\right)\right]
\end{aligned}
$$

when $D, C_{s}, C_{0}$ are constants, where $\operatorname{erf}(z)$ $=\frac{2}{\sqrt{\pi}} \int_{0}^{z} e^{-s^{2}} d s$ is the error function. But the concrete is finite in practice, numerical solutions have been concerned for a long time, for example, finite difference method in [4-7], finite element method in [8-9], and boundary element method in [10-11]

However, in the 1920s, people begin to think that the diffusion coefficient is influenced by the environment, a lot of research shows that the longer the time is, the smaller the diffusion coefficient is. Though the proof of a lot of experiments, it is considered that the diffusion coefficient can be expressed as follow [12]: 


$$
D(t)=D_{0}\left(\frac{t_{0}}{t}\right)^{\alpha}
$$

where $\alpha$ is related to the water cement ratio and affected by itself attribute of the component and the surrounding conditions. For example, $\alpha=3(0.55-w / c), D_{0}=10^{-12.06+2.4 w / c}$ and $t_{0}=28$ according to the Life-365 forecast software of the United States [13], where $w / c$ is the water cement ratio of concrete component. One-dimensional diffusion model with time varying diffusion coefficient and some modified model are discussed in [14-20].

Motivated by the above mentioned studies, we will consider a two-dimensional Chloride ion diffusing problem in a finite rectangle with time varying diffusion coefficient based on Fick's second law. In this paper, a two-dimensional ADI numerical model of Chloride diffusion is established in Section 2. At the same time, the truncation error and the stability are analyzed in Section 3. A numerical example is shown in Section 4, which can effectively predict the diffusion of Chloride ion in concrete. At last, in Section 5 the conclusion is given.

\section{Establishment of ADI Model}

Based on Fick's second law, a two -dimensional Chloride ion diffusing model in a finite rectangle with time varying diffusion coefficient is

$$
\begin{gathered}
\frac{\partial C}{\partial t}=D_{0}\left(\frac{t_{0}}{t}\right)^{\alpha}\left(\frac{\partial^{2} C}{\partial x^{2}}+\frac{\partial^{2} C}{\partial y^{2}}\right) \\
C(x, y, 0)=C_{0} \\
C(0, y, t)=C(x, 0, t)=C_{s}, \\
C\left(L_{x}, y, t\right)=C\left(x, L_{y}, t\right)=C_{0} \\
\left(0<x<L_{x}, 0<y<L_{y}\right)
\end{gathered}
$$

where $C(x, y, t)$ is the Chloride ions concentration of point $(x, y)$ at diffusion time $t, L_{x}, L_{y}$ are the length of the concrete structure, $C_{0}$ is the initial Chloride ion concentration, $C_{s}$ is the boundary Chloride ion concentration, and all of them with $D_{0}, t_{0}, \alpha$ are constants.

\subsection{Mesh}

First, the finite area $\Omega=\left\{(x, y) \mid \quad 0<x<L_{x}, 0<y<L_{y}\right\}$ and the time $[0, T]$ should be meshed, which is the outcome of discretely decomposing the continuous space. In the $x$ direction it is equally divided into $N_{x}$ parts, with $x_{i}=i h_{x}, i=0,1, \cdots, N_{x}$, and $h_{x}=L_{x} / N_{x}$ is the length of each subinterval. Similarly, in the $y$ direction it is equally divided into $N_{y}$ parts, with $y_{j}=j h_{y}, \mathrm{j}=0,1, \cdots, N_{y}$, and $h_{y}=L_{y} / N_{y}$ is the length of each subinterval. Along the time direction, select $\tau=T / N_{t}$ to be the step length, with $t_{n}=n \tau, \mathrm{n}=0,1, \cdots \cdots N_{t}$. Thus $C_{i, j}^{n}$ approximates the Chloride concentration ion $C\left(x_{i}, y_{j}, t_{n}\right)$. For simplicity, $h=h_{x}=h_{y}$.

\subsection{Discretion}

According to the above mesh and the Taylor expansion, we have

$$
\begin{gathered}
\frac{C\left(x_{i}, y_{j}, t_{n+1}\right)-C\left(x_{i}, y_{j}, t_{n}\right)}{\tau} \\
=\frac{\partial}{\partial t} C\left(x_{i}, y_{j}, t_{n}\right)+O(\tau) \\
\frac{C\left(x_{i+1}, y_{j}, t_{n}\right)-2 C\left(x_{i}, y_{j}, t_{n}\right)+C\left(x_{i-1}, y_{j}, t_{n}\right)}{h^{2}} \\
=\frac{\partial^{2}}{\partial x^{2}} C\left(x_{i}, y_{j}, t_{n}\right)+O\left(h^{2}\right) \\
\frac{C\left(x_{i}, y_{j+1}, t_{n}\right)-2 C\left(x_{i}, y_{j}, t_{n}\right)+C\left(x_{i}, y_{j-1}, t_{n}\right)}{h^{2}} \\
=\frac{\partial^{2}}{\partial y^{2}} C\left(x_{i}, y_{j}, t_{n}\right)+O\left(h^{2}\right)
\end{gathered}
$$

\subsection{ADI Model}

According to ADI method [21], when calculating the Chloride ions concentration at
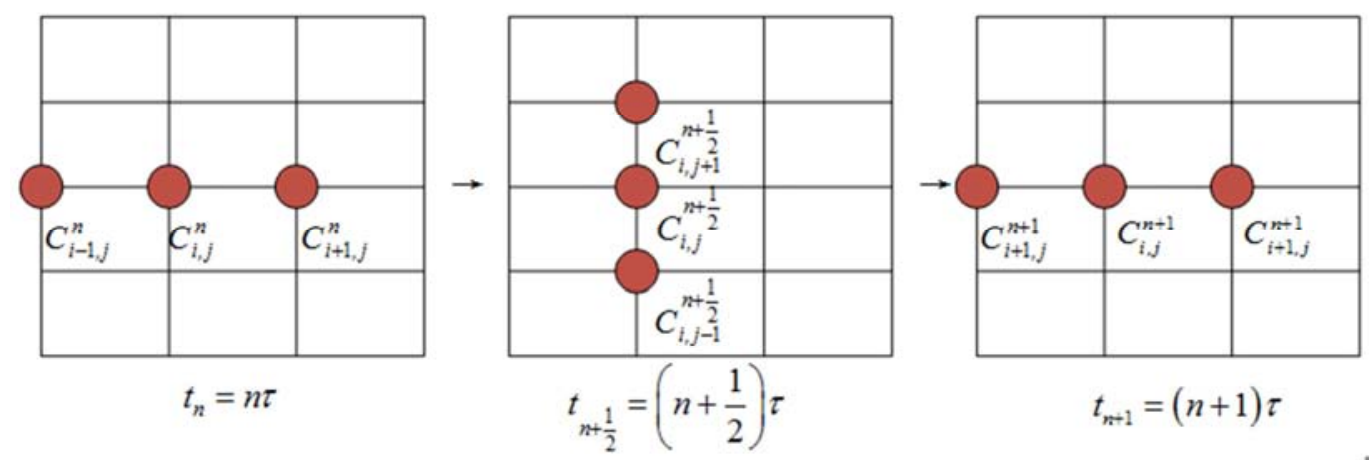

Figure 1. Two half steps of time. 
time $t_{n+1}$ by that of time $t_{n}$, a middle transition time $t_{n+\frac{1}{2}}=\left(n+\frac{1}{2}\right) \tau$ is introduced, as shown in Fig. 1.

The first step: In terms of the first half steps of time, that is, from the $n$ time layer to the $n+\frac{1}{2}$ time layer, using the implicit method to solve $\frac{\partial^{2} C}{\partial x^{2}}$, and using the explicit method to solve $\frac{\partial^{2} C}{\partial y^{2}}$, and taking $t=n \tau$, according to the equation, we can get

$$
\begin{aligned}
& \frac{C_{i, j}^{n+\frac{1}{2}}-C_{i, j}^{n}}{\tau / 2}=D_{0}\left(\frac{t_{0}}{n \tau}\right)^{\alpha} . \\
& {\left[\frac{C_{i+1, j}^{n}-2 C_{i, j}^{n}+C_{i-1, j}^{n}}{h^{2}}+\frac{C_{i, j+1}^{n+\frac{1}{2}}-2 C_{i, j}^{n+\frac{1}{2}}+C_{i, j-1}^{n+\frac{1}{2}}}{h^{2}}\right]}
\end{aligned}
$$

The second step: And at the second half steps of time, that is, from the $n+\frac{1}{2}$ time layer to the $n+1$ time layer, using the explicit method to solve $\frac{\partial^{2} C}{\partial x^{2}} \sqrt{a^{2}+b^{2}}$, and using the implicit method to solve $\frac{\partial^{2} C}{\partial y^{2}}$, and taking $t=n \tau$, according to the equation, we can get

$$
\begin{aligned}
& \frac{C_{i, j}^{n+1}-C_{i, j}^{n+\frac{1}{2}}}{\tau / 2}=D_{0}\left(\frac{t_{0}}{n \tau}\right)^{\alpha} . \\
& {\left[\frac{C_{i+1, j}^{n+1}-2 C_{i, j}^{n+1}+C_{i-1, j}^{n+1}}{h^{2}}+\frac{C_{i, j+1}^{n+\frac{1}{2}}-2 C_{i, j}^{n+\frac{1}{2}}+C_{i, j-1}^{n+\frac{1}{2}}}{h^{2}}\right],}
\end{aligned}
$$

For simplicity, assume $\lambda_{n}=\frac{\tau}{2 h^{2}} D_{0}\left(\frac{t_{0}}{n \tau}\right)^{\alpha}$, we can get the following result.

Theorem 1: The ADI model of Chloride ions diffusion with time varying coefficients is

$$
\begin{aligned}
& \left\{\begin{array}{l}
-\lambda_{n} C_{i+1, j}^{n}+\left(2 \lambda_{n}-1\right) C_{i, j}^{n}-\lambda_{n} C_{i-1, j}^{n} \\
=\lambda_{n} C_{i, j+1}^{n+\frac{1}{2}}-\left(2 \lambda_{n}+1\right) C_{i, j}^{n+\frac{1}{2}}+\lambda_{n} C_{i, j-1}^{n+\frac{1}{2}} \\
-\lambda_{n} C_{i, j+1}^{n+\frac{1}{2}}+\left(2 \lambda_{n}-1\right) C_{i, j}^{n+\frac{1}{2}}-\lambda_{n} C_{i, j-1}^{n+\frac{1}{2}} \\
=\lambda_{n} C_{i+1, j}^{n+1}-\left(2 \lambda_{n}+1\right) C_{i, j}^{n+1}+\lambda_{n} C_{i-1, j}^{n+1}
\end{array}\right. \\
& \left(i=1,2, \cdots, N_{x}-1 ; j=1,2, \cdots, N_{y}-1\right)
\end{aligned}
$$

where $\quad \lambda_{n}=\frac{\tau}{2 h^{2}} D_{0}\left(\frac{t_{0}}{n \tau}\right)^{\alpha}\left(n=0,1, \cdots N_{t}\right)$, the boundary conditions and the initial conditions are respectively

$$
\begin{aligned}
& C_{0, j}^{n}=C_{i, 0}^{n}=C_{s}, C_{N_{x}, j}^{n}=C_{i, N_{y}}^{n}=C_{0} \\
& C_{i, j}^{0}=C_{0} \quad\left(i=0,1, \cdots, N_{x} ; j=0,1, \cdots, N_{y}\right)
\end{aligned}
$$

Thus, a two-dimensional ADI numerical model of Chloride diffusion in a finite rectangle with time varying diffusion coefficient is established, that is, Eq. (12) and Eq. (13).

\section{Stability and Convergence Analysis of ADI Model}

Theorem 2: The truncation error of the ADI model (12) of Chloride ions diffusion with time varying coefficients is $O\left(a \tau^{-\alpha}+\tau^{-\alpha} h^{2}+\tau^{2-\alpha}\right)$.

Proof: According to the equations (10) and (11), simplify it and get

$$
\left\{\begin{array}{l}
\frac{C_{i, j}^{n+\frac{1}{2}}-C_{i, j}^{n}}{\tau / 2}=\frac{D_{0}}{h^{2}}\left(\frac{t_{0}}{n \tau}\right)^{\alpha}\left(\delta_{x}^{2} C_{i, j}^{n}+\delta_{y}^{2} C_{i, j}^{n+\frac{1}{2}}\right) \\
\frac{C_{i, j}^{n+1}-C_{i, j}^{n+\frac{1}{2}}}{\tau / 2}=\frac{D_{0}}{h^{2}}\left(\frac{t_{0}}{n \tau}\right)^{\alpha}\left(\delta_{x}^{2} C_{i, j}^{n+1}+\delta_{y}^{2} C_{i, j}^{n+\frac{1}{2}}\right)
\end{array} .\right.
$$

Add the above two equations, we can get

$$
\begin{aligned}
& \frac{C_{i, j}^{n+1}-C_{i, j}^{n}}{\tau / 2}=2 \frac{D_{0}}{h^{2}}\left(\frac{t_{0}}{n \tau}\right)^{\alpha} \delta_{y}^{2} C_{i, j}^{n+\frac{1}{2}} \\
& +\frac{D_{0}}{h^{2}}\left(\frac{t_{0}}{n \tau}\right)^{\alpha} \delta_{x}^{2}\left(C_{i, j}^{n+1}+C_{i, j}^{n}\right)
\end{aligned}
$$

And subtract the above two equations, we can get

$$
4 C_{i, j}^{n+\frac{1}{2}}=2\left(C_{i, j}^{n+1}+C_{i, j}^{n}\right)-\frac{\tau D_{0}}{h^{2}}\left(\frac{t_{0}}{n \tau}\right)^{\alpha} \delta_{y}^{2}\left(C_{i, j}^{n+1}+C_{i, j}^{n}\right)
$$

Substitute (16) into (15), we can get

$$
\begin{aligned}
& {\left[1+\frac{1}{4} \frac{\tau^{2} D_{0}}{h^{4}}\left(\frac{t_{0}}{n \tau}\right)^{\alpha} \delta_{x}^{2} \delta_{y}^{2}\right] \frac{C_{i, j}^{n+1}-C_{i, j}^{n}}{\tau}} \\
& =\frac{D_{0}}{h^{2}}\left(\frac{t_{0}}{n \tau}\right)^{\alpha}\left(\delta_{x}^{2}+\delta_{y}^{2}\right) \frac{C_{i, j}^{n+1}+C_{i, j}^{n}}{2}
\end{aligned}
$$

Assume $C\left(x_{i}, y_{j}, t_{n}\right)$ is the actual solution, then let $\tilde{D}=D_{0}\left(\frac{t_{0}}{n}\right)^{\alpha}$, and we can expand (17) with Taylor series, then we can get truncation error of (12). That is

$$
\left(\frac{\partial}{\partial t}+\frac{1}{2 !} \frac{\partial^{2}}{\partial t^{2}}+\cdots\right) C_{i, j}^{n}
$$




$$
\begin{aligned}
& +\left(\frac{\tau^{2-\alpha} \tilde{D}^{2}}{4} \frac{\partial^{5}}{\partial x^{2} \partial y^{2} \partial t}+\frac{\tau^{3-\alpha} \tilde{D}^{2}}{8} \frac{\partial^{6}}{\partial x^{2} \partial y^{2} \partial t^{2}}\right. \\
& \left.+\frac{h^{2} \tau^{2-\alpha} \tilde{D}^{2}}{48} \frac{\partial^{7}}{\partial x^{4} \partial y^{2} \partial t}+\frac{h^{2} \tau^{2-\alpha} \tilde{D}^{2}}{48} \frac{\partial^{7}}{\partial x^{2} \partial y^{4} \partial t}+\cdots\right) C_{i, j}^{n} \\
& -\left(\tilde{D} \tau^{-\alpha} \frac{\partial^{2}}{\partial x^{2}}+\tilde{D} \tau^{-\alpha} \frac{\partial^{2}}{\partial y^{2}}+\frac{\tau^{1-\alpha} \tilde{D}}{2} \frac{\partial^{3}}{\partial t \partial x^{2}}\right. \\
& \left.+\frac{\tau^{1-\alpha} \tilde{D}}{2} \frac{\partial^{3}}{\partial t \partial y^{2}}+\frac{\tau^{-\alpha} h^{2} \tilde{D}}{24} \frac{\partial^{4}}{\partial x^{4}}+\frac{\tau^{-\alpha} h^{2} \tilde{D}}{24} \frac{\partial^{4}}{\partial y^{4}}+\cdots\right) C_{i, j}^{n}, \\
& =\left(\tilde{D} \tau^{-\alpha} \frac{\partial^{2}}{\partial x^{2}}+\tilde{D} \tau^{-\alpha} \frac{\partial^{2}}{\partial y^{2}}+\frac{\tau^{1-\alpha} \tilde{D}}{2} \frac{\partial^{3}}{\partial t \partial x^{2}}\right. \\
& \left.+\frac{\tau^{1-\alpha} \tilde{D}}{2} \frac{\partial^{3}}{\partial t \partial y^{2}}+\frac{-\alpha \tau^{-\alpha} \tilde{D}}{2} \frac{\partial^{2}}{\partial x^{2}}+\frac{-\alpha \tau^{-\alpha} \tilde{D}}{2} \frac{\partial^{2}}{\partial y^{2}}+\cdots\right) C_{i, j}^{n} \\
& +\left(\frac{\tau^{2-\alpha} \tilde{D}^{2}}{4} \frac{\partial^{5}}{\partial x^{2} \partial y^{2} \partial t}+\frac{\tau^{3-\alpha} \tilde{D}^{2}}{8} \frac{\partial^{6}}{\partial x^{2} \partial y^{2} \partial t^{2}}\right. \\
& \left.+\frac{h^{2} \tau^{2-\alpha} \tilde{D}^{2}}{48} \frac{\partial^{7}}{\partial x^{4} \partial y^{2} \partial t}+\frac{h^{2} \tau^{2-\alpha} \tilde{D}^{2}}{48} \frac{\partial^{7}}{\partial x^{2} \partial y^{4} \partial t}+\cdots\right) C_{i, j}^{n} \\
& -\left(\tilde{D} \tau^{-\alpha} \frac{\partial^{2}}{\partial x^{2}}+\tilde{D} \tau^{-\alpha} \frac{\partial^{2}}{\partial y^{2}}+\frac{\tau^{1-\alpha} \tilde{D}}{2} \frac{\partial^{3}}{\partial t \partial x^{2}}\right. \\
& \left.+\frac{\tau^{1-\alpha} \tilde{D}}{2} \frac{\partial^{3}}{\partial t \partial y^{2}}+\frac{\tau^{-\alpha} h^{2} \tilde{D}}{24} \frac{\partial^{4}}{\partial x^{4}}+\frac{\tau^{-\alpha} h^{2} \tilde{D}}{24} \frac{\partial^{4}}{\partial y^{4}}+\cdots\right) C_{i, j}^{n}, \\
& =\left(\frac{-\alpha \tilde{D}}{2} \frac{\partial^{2}}{\partial x^{2}}+\frac{-\alpha \tilde{D}}{2} \frac{\partial^{2}}{\partial y^{2}}\right) \tau^{-\alpha} C_{i, j}^{n} \\
& -\left(\frac{\tau^{-\alpha} \tilde{D}}{24} \frac{\partial^{4}}{\partial x^{4}}+\frac{\tau^{-\alpha} \tilde{D}}{24} \frac{\partial^{4}}{\partial y^{4}} h^{2} C_{i, j}^{n}+\cdots,\right. \\
& =O\left(\alpha \tau^{-\alpha}+\tau^{-\alpha} h^{2}+\tau^{2-\alpha}\right) .
\end{aligned}
$$

So the truncation error is $O\left(\alpha \tau^{-\alpha}+\tau^{-\alpha} h^{2}+\tau^{2-\alpha}\right)$.

When $\alpha=0$, the coefficient is a constant and the truncation error is $O\left(h^{2}+\tau^{2}\right)$, which is in agreement with [7].

Theorem 3: The ADI model (12) of Chloride ions diffusion with time varying coefficients is unconditionally stable.

Proof: Let

$$
C_{j, l}^{n}=v^{n} e^{i k_{1} j h} e^{i k_{2} l h}
$$

There is growth factor $G(\tau, k, n)\left(k=\left(k_{1}, k_{2}\right)\right)$, and it satisfies

$$
C\left(j, l, t_{n+1}\right)=G(\tau, k, n) C\left(j, l, t_{n}\right)
$$

Then we can get

$$
G(\tau, k, n)=\frac{1-4 \lambda_{n} \sin ^{2} \frac{k_{1} h}{2}-4 \lambda_{n} \sin ^{2} \frac{k_{2} h}{2}}{1+4 \lambda_{n} \sin ^{2} \frac{k_{1} h}{2}+4 \lambda_{n} \sin ^{2} \frac{k_{2} h}{2}} .
$$

From the equation (12), we can get that for any $\lambda_{n}=\frac{\tau}{2 h^{2}} D_{0}\left(\frac{t_{0}}{n \tau}\right)^{\alpha}$, there is $|G(\tau, k, n)| \leq 1$, so this model is unconditionally stable.

Theorem 4: The ADI model (12) of Chloride ions diffusion with time varying coefficients is convergent.

Proof: Combine with the Lax equivalence theorem [22], we can get the ADI model of Chloride ions diffusion is consistent, according to the definition of consistency. And base on it, stability is the necessary and sufficient condition for convergence. Hence, combine with the theorem 3, we can know the differential model is convergent.

\section{Numerical Example}

Now we consider Chloride diffusion in a rectangle reinforced concrete, with $L_{y}$ is $2900 \mathrm{~mm}, L_{x}$ is $100 \mathrm{~mm}$, the Chloride ion concentration of the surface of concrete $C_{s} \sqrt{2}$ is $45 \%$ and $C_{0}$ is zero. Here take $h=1 \mathrm{~mm}$ and $\tau=1$ month . Substitute these parameters to ADI model (12) and (13), then we can predict Chloride diffusion in concrete.

(1) Different $\alpha$

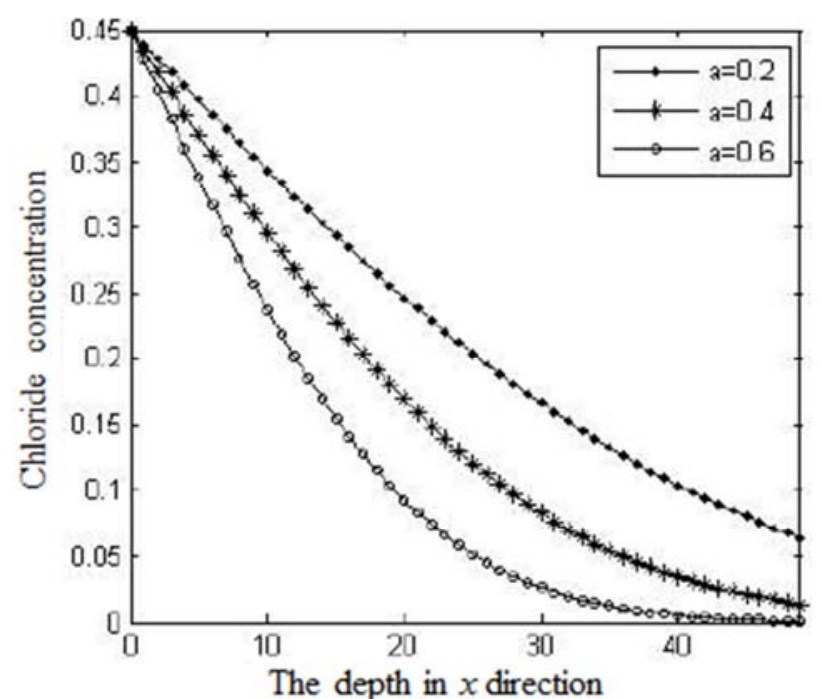

Figure 2. Chloride ion concentration with different $\alpha$.

When $T=175$ month,$D_{0}=7.17 \mathrm{~mm}^{2} /$ month , Chloride ion concentration along x-direction at $y=100 \mathrm{~mm}$ with $\alpha=0.2, \alpha=0.4, \alpha=0.6$ is shown in Fig. 2. The parameter $\alpha$ increases, the concentration decreases, which is in agreement with the property of the function $D_{0}\left(\frac{t_{0}}{t}\right)^{\alpha} \mathfrak{A}$.

(2) Different $D_{0}$

When $T=175$ month,$\alpha=0.4$, Chloride ion concentration along x-direction at $y=100 \mathrm{~mm}$ with $D_{0}=5 \mathrm{~mm}^{2} /$ month,$D_{0}=7.17 \mathrm{~mm}^{2} /$ month,

$D_{0}=10 \mathrm{~mm}^{2} /$ month is shown in Fig.3. $D_{0}$ is a fundamental diffusion coefficient, its increasing results in the acceleration of Chloride diffusion. 


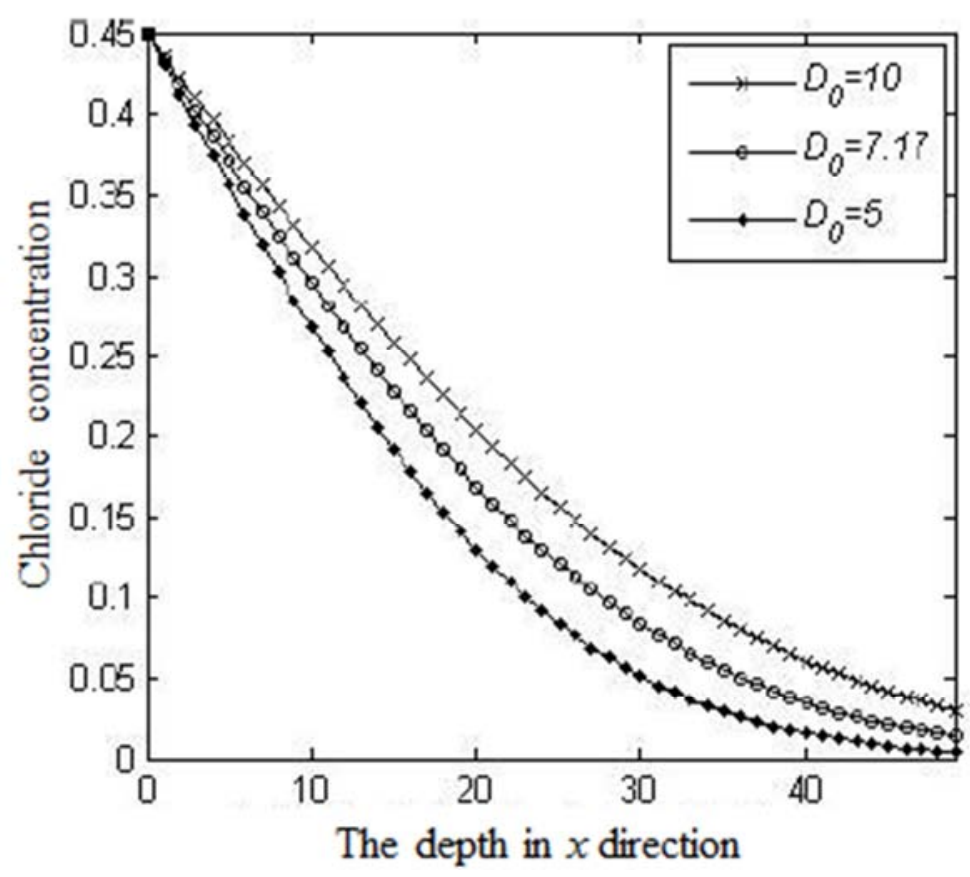

Figure 3. Chloride ion concentration with different $D_{0}$.

(3) Different $T$

When $D_{0}=7.17 \mathrm{~mm}^{2} /$ month,$\alpha=0.4$, Chloride ion concentration along x-direction at $y=100 \mathrm{~mm}$ with $T=100$ month,$T=175$ month,$T=300$ month is shown in Fig. 4.

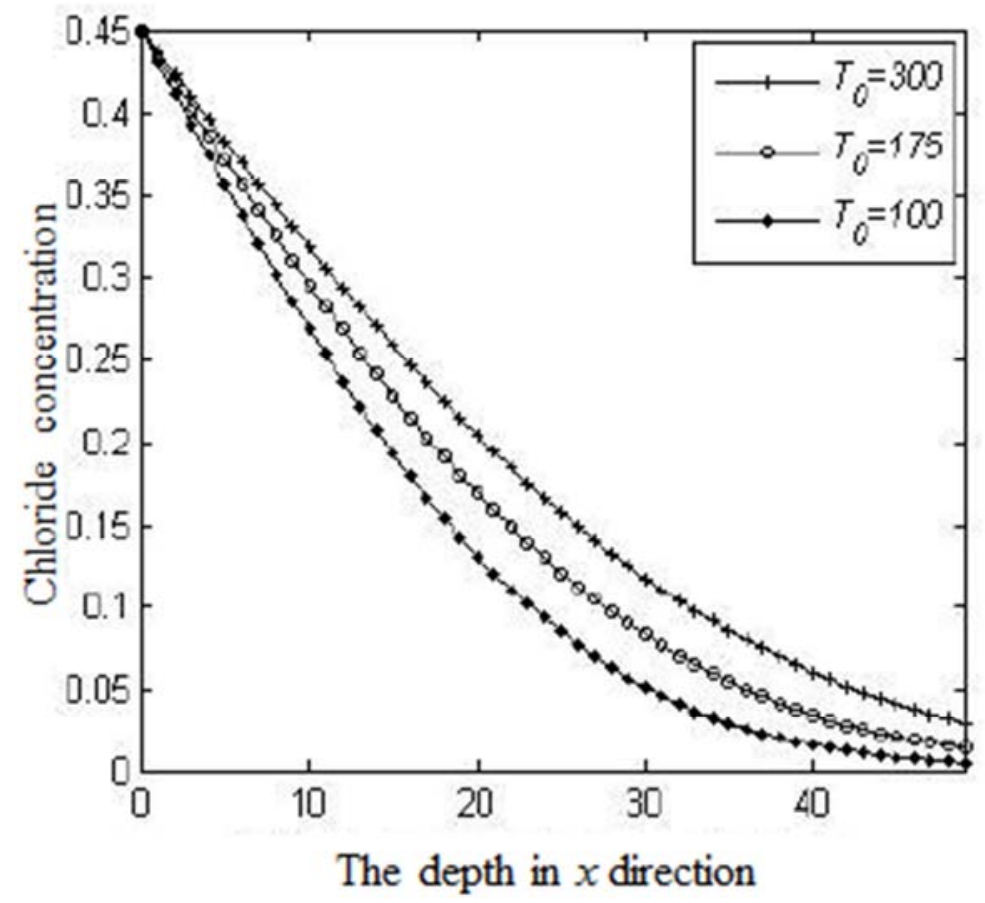

Figure 4. Chloride ion concentration with different $T$.

It is obvious that Chloride ion concentration will increase with time $T$. The larger time $T$ is, the larger Chloride ion diffusion of is, and the worse the durability of concrete.

(4) Contour Distribution

Fig. 5 shows the contour distribution of Chloride ion concentration in different situations, which describes Chloride diffusion in concrete more clearly. Figures (a) and (b) is the Chloride distribution after $T=300$ month and $T=175$ month when, $\alpha=0.6$. Figures (c) and (d) is the Chloride distribution after $T=300$ month and $T=175$ month when $D_{0}=7.17 \mathrm{~mm}^{2} /$ month,$\quad \alpha=0.2$. Figures (e) and (f) is the Chloride distribution when 
$D_{0}=10 \mathrm{~mm}^{2} /$ month and $D_{0}=7.17 \mathrm{~mm}^{2} /$ month after.

It can be seen that the increasing of the fundamental diffusion coefficient $D_{0}$ and time $T$ accelerates Chloride diffusion, while the increasing of the exponent $\alpha$ blocks Chloride diffusion. When $D_{0}=7.17 \mathrm{~mm}^{2} /$ month , $\alpha=0.6$, after $T=300$ month, that is, about 25 years, Chloride ion will diffuse to everywhere in concrete, which is very dangerous in reality.

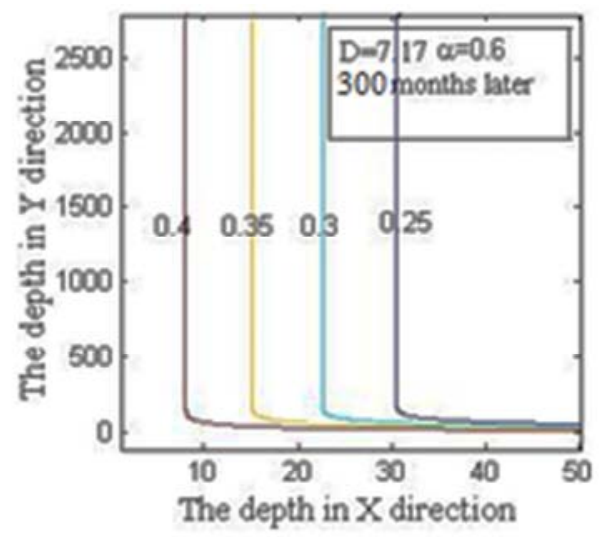

(a)

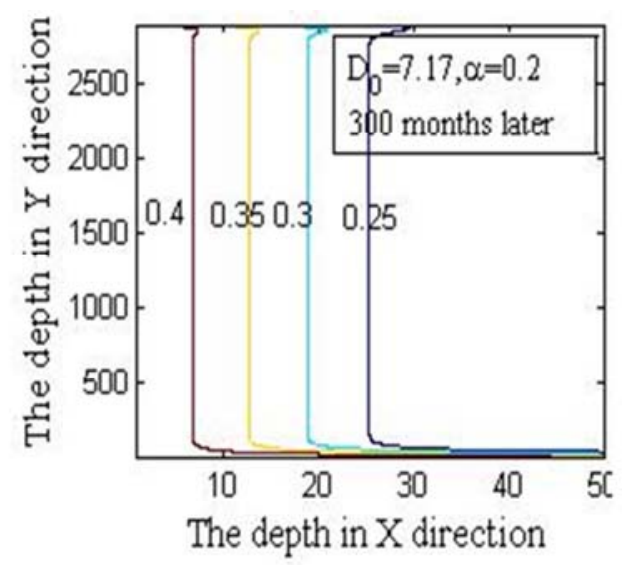

(c)

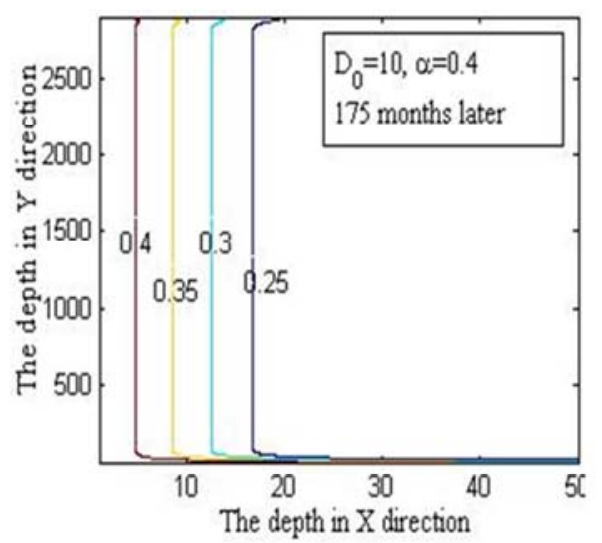

(e)

\section{Conclusion}

An ADI numerical model of two-dimensional Chloride ion diffusing problem in a finite rectangle with time varying diffusion coefficient is established, which is Eq. (12). ADI model (12) is convergent, with the truncation error $O\left(a \tau^{-\alpha}+\tau^{-\alpha} h^{2}+\tau^{2-\alpha}\right)$. And it is unconditionally stable. Numerical example shows the effectiveness of ADI model, which can predict the diffusion of Chloride ion in concrete and reflect the influence of each parameter.

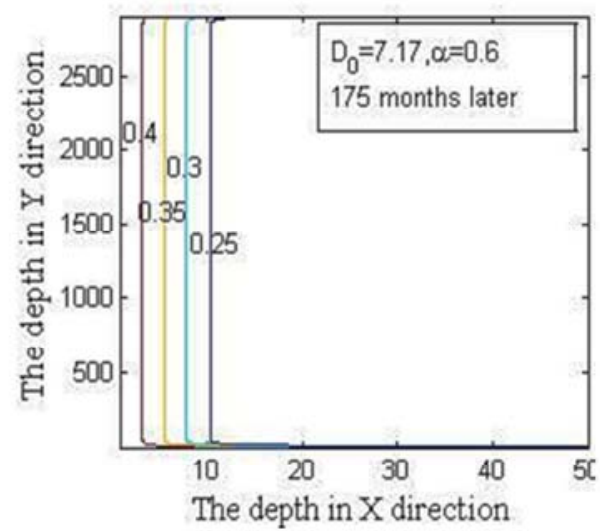

(b)

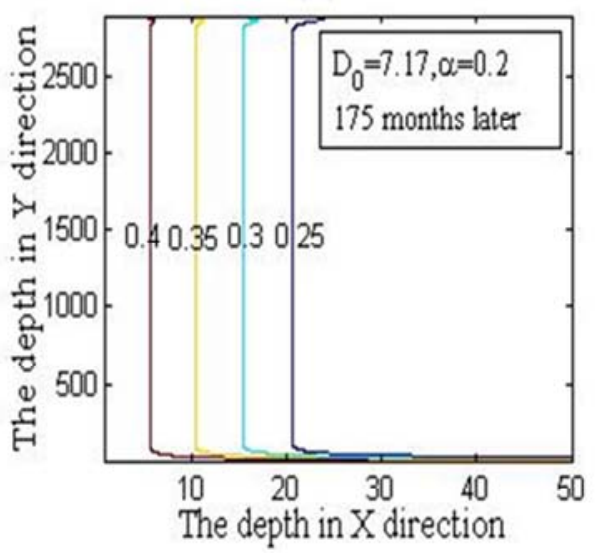

(d)

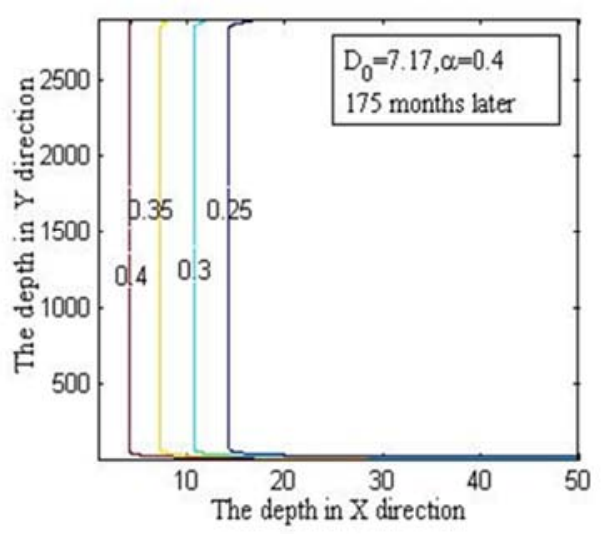

(f)

Figure 5. Contour distribution of Chloride ion concentration. 


\section{Acknowledgements}

This work is supported by the National Natural Science Foundation of China (Nos. 21206009, 21576023), the National Key Research Program of China (Nos. 2016YFC0700601, 2016 YFC070 0603), and the Science Research Foundation of Beijing University of Civil Engineering and Architecture (No. 00331616027). We express our gratitude to the editor and referees for their constructive reviews of the manuscript and helpful comments.

\section{References}

[1] Collepardi M, Marcialis A. Turrizzani R. The kinctics of penetration of Chloride ions into the concrete. II Cemenoto(Italy), 1970, 4: 157-164.

[2] Collepardi M, Marcialis A. Turrizzani R. Penetration of Chloride ions into cement pastes and concrete. 1972, 55: 534-535.

[3] Yuan, Q., Shi, C. De Schutter, G. Audenaert, K. And Deng, D. Chloride Binding of Cement-Based Materials Subjected to External Chloride Environment - A Review, Construction and Building Materials. 2009, 23 (1): 1-13.

[4] M. Funanshi. Prediction corrosion-free service life of a concrete structure in a Chloride environment. ACI Materials Journal,1990, 87 (6): 581-587.

[5] S. L. Amey, D. A. Johnson etc. Predicting the service of concrete marine structures. An Environmental Methodology, ACI Structural Journal.1998, 95 (2): 205-214.

[6] Ali H. Al-Gadhib. Numerical simulation of Chloride diffusion in RC structures and the implications of Chloride binding capacities and concrete mix. International Journal of Civil \& Environmental Engineering.2010, 10 (5): 22-35.

[7] F. Hou, Y. Bai, J. Dong. Numerical Analysis of the 2-Dimensional Diffusion Models of Chloride Ions Based on the FDM with Alternating Direction Implicit Schemes. Advanced Materials Research, 2014, 1020: 92-97.

[8] Zhang Wei, Dong Zhiliang, Lv Huang. Two-dimensional concrete Chloride ion diffusion model and engineering verification, Hydraulic Engineering. 2009, 6: 35-39.

[9] M. Bitaraf, S. Mohammadi. Analysis of Chloride diffusion in concrete structures for prediction of initiation time of corrosion using a new meshless approach. Construction and Building Materials, 2008, 22: 546-556.

[10] Lvfeng Yang, Zheng Chen etc. Numerical analysis of Chloride diffusion in concrete with time varying coefficient by boundary element method. Civil Engineering and Environmental Engineering, 2011, 33 (4): 73-79. (in Chinese).
[11] Zheng Chen, Wocheng Huang etc. Two phase boundary element model of Chloride diffusion in concrete. Concrete, 2016, 2: 12-16. (in Chinese).

[12] Hongfa Yu, Wei Sun, Haiyan Ma etc. Chloride diffusion in concrete under multiple factors. Building Material Science, 2002, 5 (3): 240-247. (in Chinese).

[13] S. H. HAN. Influence of diffusion coefficient on Chloride ion penetration of concrete structure. Construction and Building Materials, 2007, 21: 370-378. (in Chinese).

[14] E. C. Bentz. Probabilistic modeling of service life for structures subjected to Chlorides. Aci Materials Journa. 2003. 100 (5): 391-397.

[15] T. Luping, J. Gulikers. On the mathematics of time-dependent apparent Chloride diffusion coefficient in concrete. Cement and Concrete Research. 2007. 37 (4): 589-595.

[16] Y. P. Xi, Z. P. Bazant. Modeling Chloride penetration in saturated concrete. Journal of Materials in Civil Engineering, 1999, 11 (1): 58-65.

[17] N. Damrongwiriyanupap, L. Y. Li, Y. P. Xi. Coupled Diffusion of Multi-Component Chemicals in Non-Saturated Concrete. Computers and Concrete. 2013, 11 (3): 201-222.

[18] J. S. Kong, A. N. Ababneh, D. M. Frangopol, Y. P. Xi. Reliability analysis of Chloride penetration in saturated concrete. Probabilistic Engineering Mechanics. 2002, 17: 305-315.

[19] Yu Bai, Ali Harajli, Yunping Xi. Analytical Solutions of Ionic Diffusion and Heat Conduction in Multilayered Porous Media. Journal of Applied Mathematics, 2015, Article ID 208914, 11 pages, http://dx.doi.org/10.1155/2015/208914.

[20] G. M. Wang, Y. Kong, Z. H. Shui, Q. Li, J. L. Han. Experimental investigation on Chloride diffusion and binding in concrete containing metakaolin. Corrosion Engineering, Science and Technology. 2014, 49 (4): 282-286 (in Chinese).

[21] Zhang Baolin, Su Xiumin. Alternating Block Explicit-Implicit Method For the Two-Dimensional Diffusion Equation. Intern. J. Computer Math.. 1991, 38: 241-255.

[22] Lu. J. F. \& Guan. Z. Numerical solutions for partial differential equations. Tsinghua University press. 2007. (in Chinese).

[23] D. Ali, S. Mohammad. Effect of exposure temperature on Chloride-binding capacity of cementing materials. Magazine of Concrete Research. 2015, 67 (15): 821-832.

[24] Y. Ren, Q. Huang, Q. Y. Liu, J. Z. Sun, X. L. Liu. Chloride ion diffusion of structural concrete under the coupled effect of bending fatigue load and Chloride. Materials Research Innovations. 2015, 19: 181-184 (in Chinese).

[25] N. Ganesana1, A. Ruby, S. D. Rajb. Durability characteristics of steel fibre reinforced geopolymer concrete. Construction and Building Materials. 2015, 93: 471-476. 